\title{
Germline TERT promoter mutations are rare in familial melanoma
}

\author{
Mark Harland $^{1} \cdot$ Mia Petljak $^{2}$ - Carla Daniela Robles-Espinoza ${ }^{2}$ Zhihao Ding $^{2}$ • \\ Nelleke A. Gruis ${ }^{3} \cdot$ Remco van Doorn $^{3} \cdot$ Karen A. Pooley ${ }^{4}$ Alison M. Dunning ${ }^{5}$. \\ Lauren G. Aoude ${ }^{6}$ - Karin A. W. Wadt ${ }^{7}$ Anne-Marie Gerdes ${ }^{7} \cdot K^{2}$. Anin M. Brown ${ }^{8}$. \\ Nicholas K. Hayward ${ }^{6} \cdot$ Julia A. Newton-Bishop $^{1} \cdot$ David J. Adams $^{2} \cdot$ D. Timothy Bishop ${ }^{1}$
}

Published online: 3 October 2015

(c) The Author(s) 2015. This article is published with open access at Springerlink.com

\begin{abstract}
Germline $C D K N 2 A$ mutations occur in $40 \%$ of 3-or-more case melanoma families while mutations of $C D K 4, B A P 1$, and genes involved in telomere function (ACD, TERF2IP, POT1), have also been implicated in melanomagenesis. Mutation of the promoter of the telomerase reverse transcriptase (TERT) gene (c.-57 $\mathrm{T}>\mathrm{G}$ variant) has been reported in one family. We tested for the TERT promoter variant in 675 multicase families wild-type for the known high penetrance familial melanoma genes, 1863 UK population-based melanoma cases and 529 controls. Germline lymphocyte telomere length was estimated in carriers. The c. $-57 \mathrm{~T}>\mathrm{G} T E R T$ promoter variant was
\end{abstract}

Julia A. Newton-Bishop, David J. Adams, D. Timothy Bishop have contributed equally to this article.

Electronic supplementary material The online version of this article (doi:10.1007/s10689-015-9841-9) contains supplementary material, which is available to authorized users.

D. Timothy Bishop

D.T.Bishop@leeds.ac.uk

Mark Harland

m.harland@leeds.ac.uk

1 Section of Epidemiology and Biostatistics, Leeds Institute of Cancer and Pathology, University of Leeds, Leeds LS9 7TF, $\mathrm{UK}$

2 Experimental Cancer Genetics, The Wellcome Trust Sanger Institute, Hinxton, Cambridge CB10 1SA, UK

3 Department of Dermatology, Leiden University Medical Center, Leiden, The Netherlands

4 Strangeways Research Laboratory, Departments of Public Health and Primary Care, Centre for Cancer Genetic Epidemiology, University of Cambridge, 2, Worts Causeway, Cambridge CB1 8RN, UK identified in one 7-case family with multiple primaries and early age of onset (earliest, 15 years) but not among population cases or controls. One family member had multiple primary melanomas, basal cell carcinomas and a bladder tumour. The blood leukocyte telomere length of a carrier was similar to wild-type cases. We provide evidence confirming that a rare promoter variant of TERT (c. $-57 \mathrm{~T}>\mathrm{G}$ ) is associated with high penetrance, early onset melanoma and potentially other cancers, and explains $<1 \%$ of UK melanoma multicase families. The identification of POT1 and TERT germline mutations highlights the importance of telomere integrity in melanoma biology.

Keywords Melanoma - Familial - Genetic · TERT . Mutation

5 Strangeways Research Laboratory, Departments of Oncology, Centre for Cancer Genetic Epidemiology, University of Cambridge, 2, Worts Causeway, Cambridge CB1 8RN, UK

6 Oncogenomics Laboratory, QIMR Berghofer Medical Research Institute, Herston, Brisbane, QLD, Australia

7 Department of Clinical Genetics, University Hospital of Copenhagen, Copenhagen, Denmark

8 Laboratory of Translational Genomics, Division of Cancer Epidemiology and Genetics, National Cancer Institute, Bethesda, MD, USA 


\section{Background}

Cutaneous melanoma occurs predominantly in the genetically predisposed, i.e. white-skinned individuals with skin that burns easily with UV exposure, and who are prone to develop melanocytic naevi [2, 3]. Genome-wide association studies confirm that the strongest melanoma-associated single nucleotide polymorphisms (SNPs) align with these melanoma-associated risk phenotypes [4].

Clustering of melanoma in families however points to rare high-penetrance dominantly inherited mutations. The most frequently mutated high-penetrance melanoma susceptibility gene is $C D K N 2 A$ (cyclin-dependent kinase inhibitor 2A), which encodes tumour suppressors p16 and p14ARF. Worldwide, CDKN2A mutations explain predisposition in approximately $40 \%$ of families with three or more cases of melanoma, ranging from $20 \%$ in Australia to $57 \%$ in Europe [5]. The proportion of families with identifiable $C D K N 2 A$ mutations varies geographically, being lower in countries with high ambient solar ultraviolet radiation populated by fair-skinned people [5]. CDKN2A mutation carriers tend to have multiple primary melanomas, early onset melanoma and an increased risk of developing pancreatic cancer [5] and have also been shown to have an increased risk of tobacco associated cancers in respiratory and digestive tissues $[6,7]$. A very small proportion $(<1 \%)$ of families worldwide have been reported with mutations in the CDK4 (Cyclin-dependent kinase 4) gene at a key p16-binding residue [8].

Since germline $C D K N 2 A$ mutations were first identified in families there has been considerable effort to identify additional causal (i.e. high-penetrance) mutations in novel genes. The Melanoma Genetics Consortium (GenoMEL, www.genomel.org) has identified a number of genes in which mutations appear to predispose to melanoma, however the number of families in which these novel genes are involved is few. Rare families susceptible to uveal and cutaneous melanoma and non-melanoma cancers such as mesothelioma and renal cell carcinoma have also been described with inactivation of BRCA1 associated protein 1 $(B A P 1)$ [9], a gene also frequently somatically mutated in uveal melanomas [10]. More recently, rare germline mutations in the protection of telomeres 1 gene ( $P O T 1)$ were found in families with early onset and multiple primary melanomas $[11,12]$. The reported mutations abolish the binding of POT1 (part of the shelterin complex) to telomeres, leading to fragile telomeres and increased telomere length, a reported association with melanoma predisposition [13]. Mutations have also been found in other members of the shelterin complex (ACD and TER$F 2 I P$ ) in melanoma families [14]. Despite this progress, however, a high proportion (around $60 \%$ ) of families worldwide cannot be explained by predisposing mutations in known genes.

Horn et al. [1] recently identified a germline mutation in the promoter of TERT, $-57 \mathrm{bp}$ (chr5:1295161 T>G, GRCh37/hg19) from the translation start site (hereinafter referred to as c. $-57 \mathrm{~T}>\mathrm{G}$ ) co-segregating with melanoma in a 14-case family. This family was characterized by early age of onset (average 34 years) of melanoma and evidence of susceptibility to other cancers. Two melanoma cases developed ovarian cancer and a third had 5 different primary cancers other than melanoma (ovary, renal cell carcinoma, bladder, breast and lung). Further, somatic mutations within the TERT promoter, that (similarly to the familial c. $-57 \mathrm{~T}>\mathrm{G}$ variant) create a new ETS transcription factor binding site, occur in a high proportion of melanomas, supporting the important role of telomeres and TERT in melanoma [15].

The inheritance of common genetic variation that predicts telomere length is a risk factor for melanoma [16] and the identification of telomere-related genes (POT1, TERT, $A C D$ and TERF2IP) as highly penetrant susceptibility genes in melanoma families suggests that telomere regulation has considerable biological relevance in melanoma. POT1 germline carriers have longer germline telomeres than matched controls [11] there is as yet no published evidence with regard to telomere length for individuals carrying TERT promoter variants.

\section{Methods}

\section{TERT promoter analyses}

\section{Screening for mutations in melanoma families}

Melanoma families were recruited in Leeds, UK (data protection and Ethical Committee reference NIGB MR64, MREC 99/3/45), Copenhagen, Denmark (Ethics Committee of the capitol region of Copenhagen, H-3-2011-050), Brisbane, Australia (approved by QIMR Berghofer Human Research Ethics Committee) and Leiden, Netherlands (ethics approval number, P00.117).

Germline DNA from the probands of 67 UK, 16 Danish, 169 Australian, and 21 Dutch families (total of 273 families) with 3 or more cases of melanoma without known high penetrance mutations (CDKN2A, CDK4, BAP1, POT1, ACD, TERF2IP) were screened. A further 402 families consisting of 2 cases of melanoma recruited in the UK (93 families), Denmark (72 families) and Australia (237 families) were also screened.

The TERT promoter critical region (encompassing -57 to $-149 \mathrm{bp}$ ) was amplified and capillary-sequenced using 
the same primers as described in Horn et al. [1], to examine the c. $-57 \mathrm{~T}>\mathrm{G}$ germline mutation and the two most prevalent somatic mutations (c. $-124 \mathrm{G}>\mathrm{A}$ and c. -146 $\mathrm{G}>\mathrm{A}$ from the translation start site [TSS]; these mutations were termed "228" and "250" in [1] reflecting their absolute basepair location rather than as here to the TSS).

\section{Screening samples from population-based cases and controls}

In order to estimate the prevalence of germline TERT promoter mutations in population-ascertained melanoma cases, we screened blood-derived DNA from 1863 cases and 529 controls recruited to the Leeds Melanoma Case-Control study [17]. A custom Taqman ${ }^{\circledR}$ assay (Thermo Fisher Scientific; AHGJ4ZR) was used to screen these samples for the c. $-57 \mathrm{~T}>\mathrm{G}$ TERT promoter variant. We also attempted to screen the 2 main sites somatically mutated using Taqman ${ }^{\circledR}$ assays but the assay for c. $-146 \mathrm{G}>\mathrm{A}$ failed manufacture (custom assay AHHS25Z for c. $-124 \mathrm{G}>\mathrm{A}$ ).

\section{Haplotype/relatedness studies}

The $1.06 \mathrm{Mb}$ risk haplotype around the c. $-57 \mathrm{~T}>\mathrm{G}$ TERT promoter mutation contains 6 rare SNP variants in the reported family [1]. To test if the variant is recurrent or a founder mutation, we examined the segregating haplotype for these 6 SNPs, and we conducted relatedness analysis based on the Illumina HumanCytoSNP-12v2_H array, publically available SNP allele frequencies and identityby-descent analysis using PLINK [18].

\section{Telomere length assays}

Telomere length (TL) data was available from two approaches: (A) from whole exome sequence data with a method described by Ding et al. [19] and (B) using a modified q-PCR approach. For (A) germline DNA samples from melanoma cases with a family history were subject to targeted pulldown prior to next generation sequencing. The algorithm estimates telomere length by counting the offtarget telomeric repeat units and normalizing by the total number of reads with GC composition comparable to that of the telomere region $(\sim 50 \%)$. TL was estimated for all 41 familial melanoma cases for whom whole exome sequencing was conducted at the Wellcome Trust Sanger Institute; this set included samples shown to have POT1 germline mutations and previously reported showing that persons with germline POT1 mutations associated with dominantly inherited susceptibility to melanoma had increased telomere length with these assays compared to cases without POT1 mutations [11]. For (B), relative mean TL was also estimated by a $\mathrm{SYBR}^{\circledR}$ Green real-time PCR using a version of the published q-PCR protocols modified as described previously comparing population-based cases with these families [20].

\section{Results}

\section{c. -57 T $>$ G TERT promoter variant}

The c. $-57 \mathrm{~T}>\mathrm{G} T E R T$ promoter variant was identified in the proband of a single 7-case UK family and subsequently in a sibling (Fig. 1). The family was unusual within the UK in that the youngest case (not tested) was 15 years old at diagnosis, and one case had developed 7 basal cell carcinomas (an exceptionally large number for the UK) in addition to melanomas and early onset borderline bladder cancer. No case or control within the Leeds Melanoma case-control study carried the c. $-57 \mathrm{~T}>\mathrm{G}$ variant.

\section{Other promoter variants}

The quality of the sequencing did not allow detailed examination of the whole of the region but we could assess the positions $-57,-124$ and -146 from the TSS confidently. No other variants were identified at these positions. Furthermore in the case control study there was no variation shown at position -124 , a site commonly mutated somatically.

\section{Relatedness to family reported by Horn et al}

Capillary sequencing of the UK family members showed that the variant-bearing haplotype did not contain any of

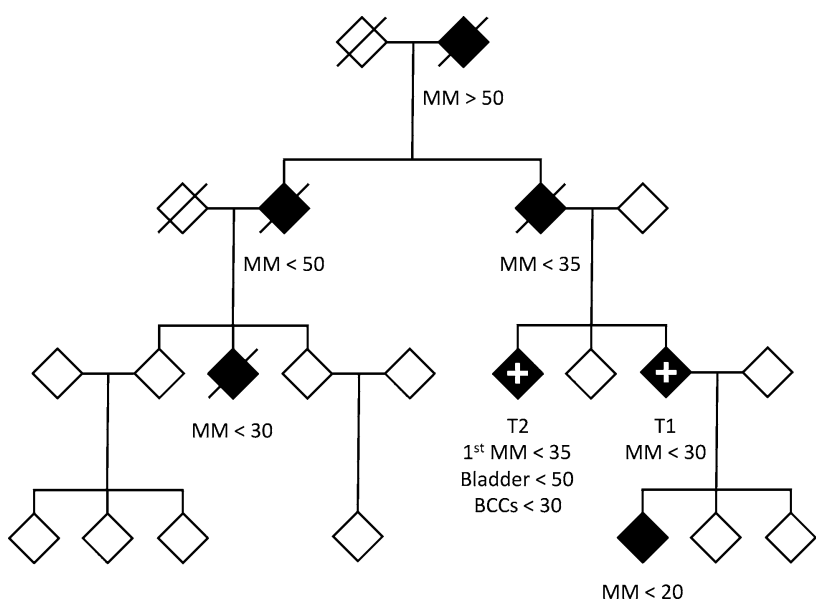

Fig. 1 Pedigree of UK family with 7 cases of melanoma in which the c. $-57 \mathrm{~T}>\mathrm{G}$ TERT promoter variant was reported in 2 affected cases. Both tested samples carried the variant allele. $M M$ shows diagnosis of first malignant melanoma. $<\mathrm{n}(>\mathrm{n})$ indicates first diagnosis before (after) age $\mathrm{n}$ years. BCC indicates basal cell carcinoma. + symbol indicates germline sample carried c. $-57 \mathrm{~T}>\mathrm{G}$ variant. Other samples were not available for testing 
the 6 rare variants seen in the reported family [1]. However, the two haplotypes do share the minor allele for rs2853669 (estimated allele frequency $=0.3$ ), which lies in the promoter $188 \mathrm{bp}$ upstream of the c. $-57 \mathrm{~T}>\mathrm{G}$ variant. To assess the significance, we examined the relatedness of the families. There was strong evidence against close relatedness of the UK family (at least 7th degree, data not shown) with the previously reported family, suggesting these are independently occurring mutations.

\section{Telomere length}

Telomere lengths were estimated by a bioinformatic analysis [19] and also by q-PCR. Because telomere length is known to be dependent on methods of DNA extraction and storage [20], this analysis was restricted to the UK families, which were processed and stored in the same laboratory as the identified family. Within the datasets there was evidence of a reduction in telomere length with age and differences by gender; however, because of sample sizes only the qPCR analysis data by age showed a significant age effect (data not shown). The bioinformatic analysis examined whole exome data from the UK multi-case melanoma families. The telomere length of the TERT promoter variant carriers was within the range shown for non-mutation carriers (Fig. 2 and Supplementary Fig. 1) ( $p=0.70$ for difference in median adjusted telomere length compared to telomeres from persons without a known germline mutation). The POT1 mutation carriers showed the longest telomeres as previously reported ( $p<0.005$ compared to persons without germline mutations) [11]. These data were previously published [11] but the figure is updated to indicate that two of the samples have subsequently been shown to have the TERT promoter mutation. In the q-PCR analysis, the telomere length of the two c. $-57 \mathrm{~T}>\mathrm{G}$ TERT variant carriers ( $\mathrm{T} 1$ and $\mathrm{T} 2$ in Fig. 1) was compared to that of 250 cases without a germline mutation but the assay repeatedly failed quality control for one of the samples (T2). T1 had an age-adjusted telomere length which was at the $10 \%$ percentile of telomere length among those without a germline mutation (i.e. had shorter telomeres than the majority of other cases) while once again POT1 mutation carriers had the longest telomere length $(\mathrm{p}<0.001$ compared to non carriers, data not shown).

\section{Discussion}

In this report, we describe the contribution of inherited TERT promoter mutations to melanoma susceptibility by screening samples from 675 melanoma families consisting of 273 with 3 or more cases and 402 with 2 cases of

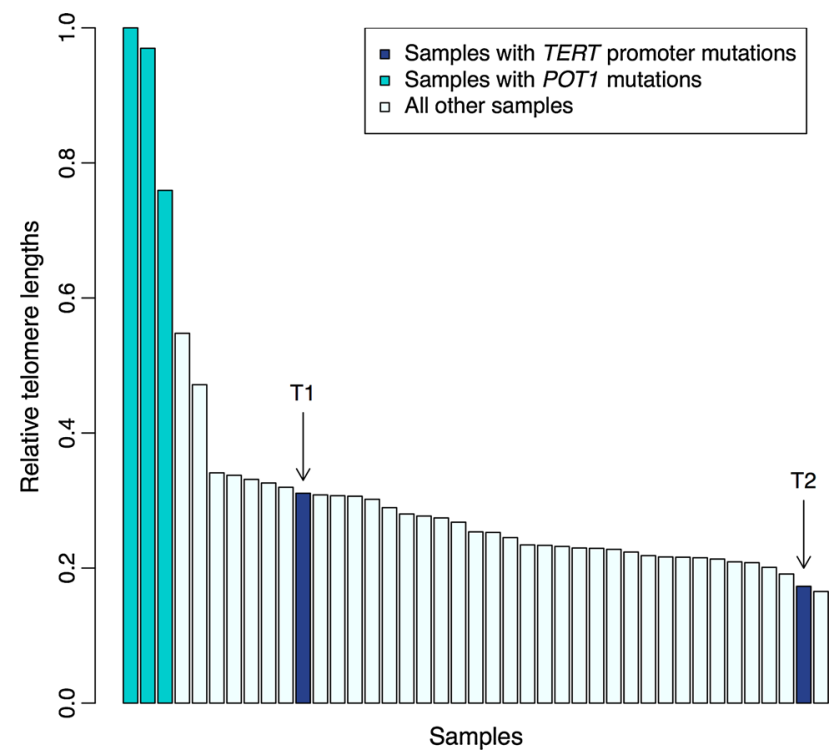

Fig. 2 Estimated telomere length of familial melanoma cases relative to the telomere length of the case with the longest telomere. Adjustment for age and sex makes minimal difference to these results because of the limited age range of those recruited so these are the raw estimates from bioinformatic analysis of whole exome resequencing data [19]. Each sample reflects the germline DNA from a melanoma case also with a family history. $\mathrm{T} 1$ and $\mathrm{T} 2$ refer to the 2 tested samples in Fig. 1 with the TERT promoter variant. This figure is modified from Robles-Espinoza et al. [11]

melanoma. We identified the previously reported c. -57 $\mathrm{T}>\mathrm{G} T E R T$ promoter variant in a single 7-case family from the UK, providing further support for this germline mutation being a very rare high penetrance melanoma susceptibility allele. This germline TERT promoter variant was identified previously by Horn et al. making this only the second family that has been identified carrying this variant worldwide. From the size of our study and the lack of other families with this mutation in the initial study, it is clear that this inherited mutation is extremely rare in melanoma families. A strength of this report is that we have screened a substantial number of melanoma families from Australia, Denmark, the Netherlands and the UK.

While a detailed assessment of the contribution of this variant to melanoma risk is not feasible, we can put these findings in some context. The UK family with the promoter variant was identified came from a series of 139 three-ormore case melanoma families, of which 56 (40\%) have a CDKN2A mutation; 2 (1.4\%) have a CDK4 mutation, 4 $(2.8 \%)$ have shelterin mutations, and now $1(0.7 \%)$ has a TERT promoter mutation.

In the two TERT mutation positive families, there are a large number of melanoma cases and other cancers. Both families had mutation carriers with bladder cancer. It is of interest that analyses from The Cancer Genome Atlas (TCGA) identified the TERT promoter to be a somatic mutation hotspot for melanoma and bladder cancer as well 
as glioblastoma, glioma, liver, medulloblastoma and lung cancer [21]. It is speculative but TERT could predispose to a broader range of cancers than melanoma.

Small numbers preclude a statistical analysis of the numbers of naevi and atypical naevi in POTI and TERT mutation carriers but the POT1 mutation carriers have a number of atypical naevi in the top $10 \%$ of atypical naevi among our cohort of melanoma cases (data not shown). This might be related to factors above and beyond the presence of the POT1 mutation (e.g. behaviour in the sun); a much larger study is required to make more informed comments about the naevus phenotype.

TERT encodes the enzyme telomerase reverse transcriptase, a subunit of telomerase, which maintains telomere ends by addition of the repeat TTAGGG. Overexpression of telomerase is key component of the transformation process in many malignant cancer cells. The c.57 $\mathrm{T}>\mathrm{G}$ mutation is hypothesised to create an ETS transcription factor binding site in the TERT promoter and so enhance transcription of the TERT gene [1]. The rs2853669 polymorphism (at c.-246) detected in conjunction with c. $-57 \mathrm{~T}>\mathrm{G}$ in both $\mathrm{UK}$ and reported samples has also been reported to disrupt an ETS binding site and was associated with low telomerase activity in patients with non small cell lung cancer [22]. The loss of this ETS binding site might be expected to moderate the effect of the ETS site created by the mutation at c. -57 , however the combination of both rs 2853669 and c. $-57 \mathrm{~T}>\mathrm{G}$ appears to result in a greater increase in promoter activity than the presence of c. -57 $\mathrm{T}>\mathrm{G}$ alone [1].

Recently, predicted telomere length (based upon inheritance of SNPs identified in genome-wide association studies to be associated with telomere length) was reported to be a risk factor for melanoma [16]. That predicted telomere score has been established as a strong risk factor for melanoma in the general population [16], and germline POT1, TERT and other shelterin complex gene mutations have been found in melanoma families, suggests that telomere function is critical in melanoma susceptibility. In this study we did not demonstrate increased telomere length in the lymphocyte of TERT promoter mutation carriers, in contrast to our recent study in which we did see long telomeres in families with inherited mutations in POT1 [11]. The implication is that the TERT promoter mutation may disrupt the function of the telomere in ways other than by simply increasing its length, as evidenced by findings of common variants that affect cancer risk but not directly via telomere length [20] Alternatively the effect on telomere length may be tissue-specific and the effect differs between melanocytic and lymphocytic.

Acknowledgments The authors would like to thank the families for their willingness to participate; and Rajiv Kumar for the provision of mutation positive samples. The collection of samples in the UK population-ascertained sample set was funded by Cancer Research UK (awards C588/A19167 and C8216/A6129) and by the NIH (CA83115). The work of N.A.G. and R.v.D was supported by the Dutch Cancer Society (UL 2012-5489). D.J.A and C.D.R.E are supported by Cancer Research UK, ERC Combat Cancer and the Wellcome Trust. N.K.H is supported by a fellowship from the National Health and Medical Research Council of Australia. A.M.D. and K.A.P. were supported by CRUK grant (C8197/A16565) and The Isaac Newton Trust. K.M.B. is supported by the Intramural Research Program of the Division of Cancer Epidemiology and Genetics; National Cancer Institute; National Institutes of Health.

\section{Compliance with ethical standards}

Conflict of interest The authors declare that they have no conflict of interest.

Open Access This article is distributed under the terms of the Creative Commons Attribution 4.0 International License (http://creative commons.org/licenses/by/4.0/), which permits unrestricted use, distribution, and reproduction in any medium, provided you give appropriate credit to the original author(s) and the source, provide a link to the Creative Commons license, and indicate if changes were made.

\section{References}

1. Horn S, Figl A, Rachakonda PS et al (2013) TERT promoter mutations in familial and sporadic melanoma. Science 339(6122):959-961

2. Gandini S, Sera F, Cattaruzza MS et al (2005) Meta-analysis of risk factors for cutaneous melanoma: III. Family history, actinic damage and phenotypic factors. Eur J Cancer 41(14):2040-2059

3. Gandini S, Sera F, Cattaruzza MS et al (2005) Meta-analysis of risk factors for cutaneous melanoma: I. Common and atypical naevi. Eur J Cancer 41(1):28-44

4. Barrett JH, Iles MM, Harland M et al (2011) Genome-wide association study identifies three new melanoma susceptibility loci. Nat Genet 43(11):1108-1113

5. Goldstein AM, Chan M, Harland M et al (2007) Features associated with germline CDKN2A mutations: a GenoMEL study of melanoma-prone families from three continents. J Med Genet 44(2):99-106

6. Helgadottir H, Höiom V, Jönsson G et al (2014) High risk of tobacco-related cancers in CDKN2A mutation-positive melanoma families. J Med Genet 51(8):545-552

7. Potrony M, Puig-Butillé JA, Aguilera P et al (2014) Increased prevalence of lung, breast, and pancreatic cancers in addition to melanoma risk in families bearing the cyclin-dependent kinase inhibitor 2A mutation: implications for genetic counseling. J Am Acad Dermatol 71(5):888-895

8. Puntervoll HE, Yang XR, Vetti HH et al (2013) Melanoma prone families with CDK4 germline mutation: phenotypic profile and associations with MC1R variants. J Med Genet 50(4):264-270

9. Carbone M, Ferris LK, Baumann F et al (2012) BAP1 cancer syndrome: malignant mesothelioma, uveal and cutaneous melanoma, and MBAITs. J Transl Med 10:179

10. Harbour JW, Onken MD, Roberson ED et al (2010) Frequent mutation of BAP1 in metastasizing uveal melanomas. Science 330(6009):1410-1413

11. Robles-Espinoza CD, Harland M, Ramsay AJ et al (2014) POT1 loss-of-function variants predispose to familial melanoma. Nat Genet 46(5):478-481 
12. Shi J, Yang XR, Ballew B et al (2014) Rare missense variants in POT1 predispose to familial cutaneous malignant melanoma. Nat Genet 46(5):482-486

13. Burke LS, Hyland PL, Pfeiffer RM et al (2013) Telomere length and the risk of cutaneous malignant melanoma in melanomaprone families with and without CDKN2A mutations. PLoS One 8(8):e71121

14. Aoude LG, Pritchard AL, Robles-Espinoza CD et al (2015) Nonsense mutations in the shelterin complex genes ACD and TERF2IP in familial melanoma. J Natl Cancer Inst 107(2):dju408

15. Huang FW, Hodis E, Xu MJ, Kryukov GV, Chin L, Garraway LA (2013) Highly recurrent TERT promoter mutations in human melanoma. Science 339(6122):957-959

16. Iles MM, Bishop DT, Taylor JC et al (2014) The effect on melanoma risk of genes previously associated with telomere length. J Natl Cancer Inst 106(10):dju267

17. Newton-Bishop JA, Chang YM, Iles MM et al (2010) Melanocytic nevi, nevus genes, and melanoma risk in a large case- control study in the United Kingdom. Cancer Epidemiol Biomark Prev 19(8):2043-2054

18. Purcell S, Chang C. PLINK 1.9. Secondary PLINK 1.9. http:// www.cog-genomics.org/plink2

19. Ding Z, Mangino M, Aviv A, Spector T, Durbin R, Consortium UK (2014) Estimating telomere length from whole genome sequence data. Nucleic Acids Res 42(9):e75

20. Pooley KA, Sandhu MS, Tyrer J et al (2010) Telomere length in prospective and retrospective cancer case-control studies. Cancer Res 70(8):3170-3176

21. Weinhold N, Jacobsen A, Schultz N, Sander C, Lee W (2014) Genome-wide analysis of noncoding regulatory mutations in cancer. Nat Genet 46(11):1160-1165

22. Hsu CP, Hsu NY, Lee LW, Ko JL (2006) Ets2 binding site single nucleotide polymorphism at the hTERT gene promoter-effect on telomerase expression and telomere length maintenance in nonsmall cell lung cancer. Eur J Cancer 42(10):1466-1474 